\title{
Structured Argumentation in a Mediator for Online Dispute Resolution
}

\author{
Ioan Alfred Letia ${ }^{1}$ and Adrian Groza ${ }^{1}$ \\ Technical University of Cluj-Napoca \\ Department of Computer Science \\ Baritiu 28, RO-400391 Cluj-Napoca, Romania \\ \{letia, adrian\}@cs-gw .utcluj.ro
}

\begin{abstract}
Online dispute resolution is becoming the main method when dealing with a conflict in e-commerce. A family of defeasible reasoning patterns is used to provide a useful link between dispute resolution agents and legal doctrines. The proposed argumentation framework combines defeasible logic with temporal reasoning and argumentation with level of certainty. The evaluation of arguments depends on the stage of the dispute: commencement, discovery, pre-trial, arbitration, according to current practice in law. By applying the open world assumption to the rules, the argumentative semantics of defeasible logic is enriched with three types of negated rules which offer symmetrical means of argumentation for both disputants. A corollary of this extension consists in defining a specialized type of undercutting defeater. The theory is illustrated with the help of a concrete business-to-client case in a partially implemented system.
\end{abstract}

\section{Introduction}

Online Dispute Resolution (ODR) promises to become the predominant approach to settle e-commerce disputes. To reach this statute it needed ten years of fast and sustained development [1]: starting in 1996 as a hobby, an experimental stage sustained by academics and non-profit organizations during 1997-1998, an entrepreneurial stage from 1999 (75\% rate of success as business), and beginning with 2003 there have been much governmental effort and many projects to institutionalize the online dispute resolution process.

Regarding the automation of the ODR process, one goal of this paper is to provide a flexible argumentation framework, according to the current practice in law, which can be effectively employed in online dispute resolution agents. In recent years several researchers acknowledged the value of argumentation theory for ODR [2]. Flexibility in configuring ODR systems is both an opportunity and a challenge. The opportunity is that any business can, quite quickly, have its own "court" specialized in disputes that might occur in its specific business domain. The challenge is that the technical instrumentation must simultaneously satisfy the business viewpoint asking for trust [3] and the legal viewpoint, which requires accordance with the current practice in law. 


\section{Argumentation Framework}

We enrich the defeasible logic of Governatori [4] with interval-based temporal reasoning and its argumentation semantics with level of certainty and negated rules. Although defeasible logic has already been proved suitable for legal reasoning [5], by introducing interval-based reasoning we attempt to provide a more appropriate framework for practical scenarios, having the possibility to model contract deadlines. The levels of certainty for weighting arguments are meant to better handle incomplete information, vagueness, or fuzziness of the terms implied in the dispute.

Definition. A theory in temporal defeasible logic (TDL) is a structure $\langle F, R\rangle$ formed by a finite set of facts $f(\beta)[a, b] \in F$ valid at time $t, a \leq t \leq b$, and $a$ finite set of rules $r(\gamma) \in R$, with certainty factors $\beta, \gamma \in(0 . .1]$. A fact $f(\beta) \in F$ is strict if $\beta=1$ and defeasible if $\beta<1$.

The rules are split in two disjoint sets: the set of support rules $R_{\text {sup }}$ which can be used to infer conclusions and the set of defeaters $R_{\text {def }}$ that can be used only to block the derivation of some conclusions.

Definition. A rule $r(\gamma) \in R_{\text {sup }}$ is strict $(\rightarrow)$ iff $\gamma=1$, with the set of strict rules $R_{s}=\left\{r(\gamma) \in R_{\text {sup }} \mid \gamma=1\right\}$. A rule $r(\gamma) \in R_{\text {sup }}$ is defeasible $(\Rightarrow)$ iff $\gamma<1$, with the set of defeasible rules $R_{d}=\left\{r(\gamma) \in R_{\text {sup }} \mid \gamma<1\right\}$.

Strict rules are rules in the classical sense, that is whenever the premises are indisputable, then so is the conclusion, while defeasible rules are rules that can be defeated by contrary evidence. Following Pollock's terminology [6], a defeasible conclusion $q$ can be defeated either by inferring the opposite one $\sim q$ with a superior certainty factor (rebuttal defeater), or by attacking $(\rightsquigarrow q)$ the link between the premises and the conclusion $q$ (undercutting defeater ${ }^{1}$ ).

Facts within TDL are enhanced with validity intervals. For premise $a[x, y]$ and a conclusion $b[u, v]$ the following weak semantics is used: if $a$ is valid in at least one moment within $[x, y]$, then $b$ is valid in all moments from $[u, v]$. In this interpretation (imprecise premise, precise conclusion), the validity interval $[\mathrm{a}, \mathrm{b}]$ of a rule depends on the activation intervals of its own premises: $r_{i}(\gamma)[a, b]: q_{1}\left(\beta_{1}\right)\left[a_{1}, b_{1}\right], \ldots q_{k}\left(\beta_{k}\right)\left[a_{k}, b_{k}\right] \Rightarrow q_{0}\left(\beta_{0}\right)\left[a_{0}, b_{0}\right]$, with $a=\min \left(a_{i}\right)$ and $b=\max \left(b_{i}\right), i \in[1 . . k]$. For the particular case when a defeasible rule has only one premise, its activation interval is synonym to the validity of that premise: $q_{1}\left[a_{1}, b_{1}\right] \Rightarrow q_{0}\left[a_{0}, b_{0}\right] \Leftrightarrow\left(q_{1} \Rightarrow q_{0}\left[a_{0}, b_{0}\right]\right)\left[a_{1}, b_{1}\right]$. This feature is used in nested rules $^{2}$.

\footnotetext{
${ }^{1}$ Intuitively, an undercutting defeater argues that the conclusion is not sufficiently supported by its premises.

${ }^{2}$ In our approach, rules are allowed to appear as premises or conclusions within other rules. The general case of such nested rule is represented by: $r_{i}\left(\gamma_{i}\right): r_{j}\left[a_{2}, b_{2}\right] \Rightarrow$ $r_{k}\left[a_{3}, b_{3}\right]$, where the existence of the rule $r_{j}$ fires the conclusion $r_{k}$, which can be seen as a dynamic rule. Another technical approach [7] consists in using an objectivation operator to translate a meta-level expression to an object-level expression.
} 
Similar to facts, the rules acting as premises or conclusion within the body of a nested rule can appear negated. We use the following notations: $\nrightarrow$ for $\neg(a \rightarrow b)$, meaning that " $a$ does not strictly determine $b$ " $\nRightarrow$ for $\neg(a \Rightarrow b)$, meaning that " $a$ does not defeasibly determine $b$ ", and $\Varangle$ for $\neg(a \rightsquigarrow b)$ meaning that " $a$ does not defeat $b$ ". We note by $R_{n s}$ the set of negated strict rules, by $R_{n d}$ the set of negated defeasible rules, and by $R_{\text {ndef }}$ the set of negated defeaters. The problem consists in giving a proper interpretation to a negated rule. Firstly, the negated rule represents a counterargument to the opposite rule, negated rules having the same role as an undercutting defeater, attacking the links between the premises and the conclusion. The difference consists in the fact that a defeater of the consequent $q$ attacks all rules which sustain $q$, whilst the negated rule attacks a single rule sustaining the respective conclusion ${ }^{3}$. The version of Toulmin's standard example

Claim: Harry is a British subject now.

Datum: Harry was born in Bermuda in 1937.

Harry is become an American citizen[1966,1966].

Very probably Harry speaks English.

Warrant: A man born in Bermuda will generally be a British subject. English speakers are usually British subject.

Backing: Civil Code 123 provides that persons born in Bermuda are generally British subjects.

Exception: An American Citizen cannot be a British subject.

Counter-example: Speaking English does not mean one is a British subject.

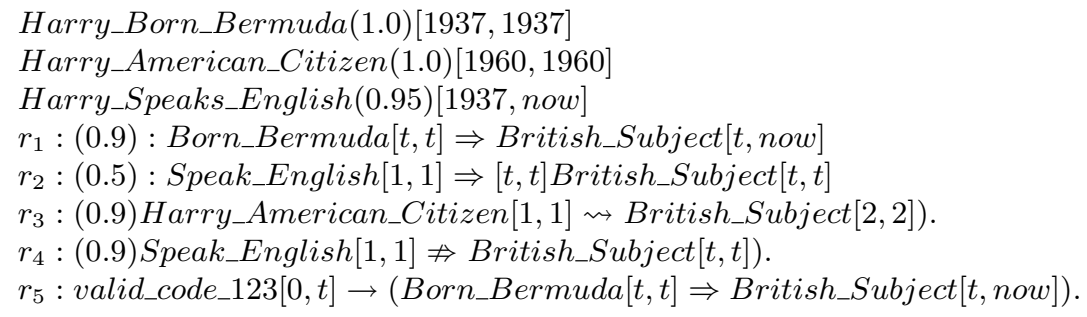

Fig. 1. A special type of undercutting defeater: negated rules.

about British citizenship in figure 1 illustrates this difference. Here, the rule $r_{4}$ attacks only the rule $r_{2}$, which is defeated. Opposite, the undercutting defeater $r_{3}$ attacks both $r_{1}$ and $r_{2}$ with a stronger certainty factor, blocking the claim $+\partial$ British_Subject : now. We use Pollock's undercutting defeaters to model exceptions and negated rules in representing counter-examples. Undercutting defeaters or negated rules cannot be used to draw a conclusion, their only use is to prevent some conclusions. Practically, introducing negated rules, we extend the open world assumption to the rules. A relation between two terms $a$ and

\footnotetext{
${ }^{3}$ If defeaters represent rules used to block the derivation of some conclusion $q$, the
} negated rules are used to block the activation of a specific support argument for $q$. 


\begin{tabular}{|c|c|}
\hline$\varphi$ & $\sim \varphi$ \\
\hline$q$ & $\neg q, X \rightarrow \neg q, X \Rightarrow \neg q$ \\
\hline$A \rightarrow q$ & $\neg q, X \rightarrow \neg q, A \nrightarrow q$ \\
\hline$A \Rightarrow q$ & $\neg q, X \rightarrow \neg q, X \Rightarrow \neg q, X \rightsquigarrow \neg q, A \nRightarrow q$ \\
\hline$A \rightsquigarrow q$ & $A \not \neg q$ \\
\hline$A \nrightarrow q$ & $A \nrightarrow q$ \\
\hline$A \nRightarrow q$ & $A \Rightarrow q$ \\
\hline$A \not \mapsto q$ & $A \rightsquigarrow q$ \\
\hline
\end{tabular}

Fig. 2. Attacking a sentence $\varphi$ depends on its type.

$b$ can be positive $(a \rightarrow b)$, negative $(a \nrightarrow b)$, or unspecified. Pairs of relations provide symmetrical means of argumentation for both disputants. The type of counterargument depends on the type of the current sentence $\varphi$ : fact, support rule, defeater (figure 2). Here, one can see that the support rules $(\rightarrow, \Rightarrow$ ) can be attacked in different ways. The negated rule $A \not \sim q$ represents an argument in favor of $q$, because it attacks the undercutting defeater $A \rightsquigarrow q$. The second utility of the negated rules is the dynamic elimination of some arguments from the knowledge base. The existence of a negated rule allows the deactivation of a rule, when the certainty factor is strong enough.

\section{Types of Agents for ODR}

A family of defeasible reasoning patterns is discussed next, employed in dispute resolution agents for more flexibility of the decision. The strategy of an agent consists of three orthogonal components which modularly capture different concerns of the problem: basic component, tactical component, and attitude component.

\subsection{Basic Component}

Fuzzy Inference. Using the weakest link principle for deductive arguments [6], the conclusion $q_{0}$ is as good as the weakest premise, given by $\min \left(\beta_{1}, \ldots, \beta_{k}\right)$. Additionally, the certainty factor is also influenced by the strength $\gamma$ of the inferencing rule (figure 3). The figure presents the generalized modus ponens where given the premises $q_{i}\left(\beta_{i}\right)\left[t_{i}\right]$ valid at time $t_{i}$ required by the rule $r_{i}$, the conclusion $q_{0}$ is inferred with a strength equal to the minimum between the strength of the premises $\beta_{i}$ and the strength of the rule $r_{i}$.

Probabilistic inference. A probabilistic approach of computing the certainty factor of a conclusion would multiply the certainty factors of all premises. Practically, the certainty factor depends on the number of premises. In this probabilistic context, the temporal persistence issue can also be considered. Suppose the fact $a$ having the certainty factor $\beta_{a}$ is valid at time $t$. The following interpretation could arise: if $a$ at $t$ then there is a defeasible reason to infer $a$ at $t+\Delta t$, the 


$$
\begin{aligned}
& \text { rule_r } r_{i}:{ }_{-} q_{0}\left[a_{0}, b_{0}\right] \stackrel{\gamma}{\longleftarrow} q_{1}\left[a_{1}, b_{1}\right] \wedge \ldots \ldots \ldots \ldots \ldots \ldots . \wedge q_{k}\left[a_{k}, b_{k}\right] \\
& \text { facts }:{ }_{-} q_{1}\left(\beta_{1}\right)\left[t_{1}\right], a_{1} \leq t_{1} \leq b_{1} \ldots \ldots \ldots \ldots \ldots \ldots, q_{k}\left(\beta_{k}\right)\left[t_{k}\right], a_{k} \leq t_{k} \leq b_{k} \\
& q_{0}\left(\min \left(\beta_{1}, \ldots \ldots, \beta_{k}, \gamma\right)\left[a_{i}\right], \forall a_{i}, a_{0} \leq a_{i} \leq b_{0}\right.
\end{aligned}
$$

Fig. 3. Inferring the conclusion $q_{0}$ when no valid defeaters exist.

certainty factor for $a$ being a monotonic decreasing function of argument $\Delta t$. A typical scenario might be: the probability that the new business partner will breach the contract is 0.2 . This probability decreases as time passes and the contract meets its time of maturity. Similarly, an agent believes that his business partner is trust-able with a factor of 0.6. If nothing defeats this believe in time, the agent increases the trust in the partnership as the business runs. By default we consider that the certainty factor is constant in time and we provide mechanisms to adjust it for each scenario.

\subsection{Tactical Component}

The same conclusion $q$ can be sustained by several arguments with different degrees of reliance. The tactical component defines how an agent handles the accrual of such valid arguments. Let $n$ be the number of valid derivations of the consequent $q$ and $c f\left[q_{i}\right]$ the certainty factor of the inference number $i$ of $q, i \in[1 . . n]$. Similarly, $m$ is the number of valid undercutting defeaters (both defeaters and negated rules) of the sentence $q$ and we note by $c f\left[\sim q_{j}\right]$ the certainty factor of the $j$ defeater of $q, j \in[1 . . m]$. If $p$ is the number of valid rebuttal defeaters, we note with $c f\left[\neg q_{k}\right]$ the certainty factor of the $k$ rebuttal defeater for $q, k \in[1 . . p]$.

Persuasion Agent. In some situations, independent reasons supporting the same action provide stronger arguments in favor of that conclusion. For instance, the testimony of two witnesses is required in judicial cases. This approach is appropriate for practical reasoning, when the decision is about what actions to perform [6] or evidential reasoning [8]. One issue related to this agent regards the difficulty to identify independent reasons. Thus, an argument presented in different forms contributes with all its avatars to the certainty factor. Similarly, an argument subsumed by another general argument also contributes to the certainty factor. Correlated to the same judicial example, if the two witnesses are kin or they conferred with each other, only one testimony is accepted in the trial. The accrual of dependent arguments is not necessarily useless. Changing the perspective, this case can be valuable in persuasion dialogs, where an agent, by repeatedly posting the same argument in different representations, will end in convincing his partner to accept that sentence.

A persuasion agent computes the certainty factor of the thesis $q$ under dispute as follows. Firstly, it considers all the accepted arguments supporting the claim 
$q$ at time $t$. This amount is decreasing by all his objections about deriving $q$, in our case all the undercutting defeaters. If the remaining certainty factor is still greater than all the existing support for the opposite conclusion $\neg q$, the thesis is successfully established. Formally, the model of persuasion based on the defeasible pattern of inference becomes:

$$
c f[q]= \begin{cases}\min \left(1, \sum_{i=1}^{n} c f\left[q_{i}\right]-\sum_{j=1}^{m} c f\left[\sim q_{j}\right]\right), & \sum_{i=1}^{n} c f\left[q_{i}\right]-\sum_{j=1}^{m} c f\left[\sim q_{j}\right]>\sum_{k=1}^{p} c f\left[\neg q_{k}\right] \\ 0, & \text { otherwise }\end{cases}
$$

Epistemic Agent. In reasoning about what to believe or epistemic reasoning the accrual of arguments does not hold [6]. The sentence $q$ is inferred if it has a greater support than any of the undercutter or rebuttal defeaters, but the certainty factor is not diminished:

$$
c f[q]= \begin{cases}\max \left(c f\left[q_{i}\right]\right), & \max \left(c f\left[q_{i}\right]\right)>\max \left(c f\left[\neg q_{k}\right], c f\left[\sim q_{j}\right]\right) \\ 0, & \text { otherwise }\end{cases}
$$

The choice between a persuasion or an epistemic agent depends on the context. A hybrid agent would include modalities such as action or knowledge for capturing practical and, respectively, epistemic reasoning, with the certainty factor of the conclusion computed accordingly.

Rigorous Agent. A rigorous agent will treat differently each type of defeater. Thus, only the strongest undercutting defeater contributes to the decreasing of the certainty factor. If the remaining strength of the conclusion overwhelms the most powerful rebuttal defeater, the respective conclusion is derived.

$$
c f[q]= \begin{cases}\max \left(c f\left[q_{i}\right]\right)-\max \left(c f\left[\sim q_{j}\right],\right. & \max \left(c f\left[q_{i}\right]\right)-\max \left(c f\left[\sim q_{j}\right]\right) \\ & >\max \left(c f\left[\neg q_{k}\right]\right) \\ 0, & \text { otherwise }\end{cases}
$$

Next we present the derivation formula of a consequent according to the reasoning strategy of the rigorous agent. A conclusion in TDL is a tagged literal which can have the following forms: i) $+\Delta q: t \Leftrightarrow q$ is definitely provable at time $t$ in $T D L$, using only strict facts and rules (figure 4); ii) $-\Delta q: t \Leftrightarrow q$ is not definitely provable at time $t$ in $T D L$; iii) $+\partial q: t \Leftrightarrow q$ is defeasibly provable at time $t$ in $T D L$ (figure5); iv) $-\partial q: t \Leftrightarrow q$ is not defeasibly provable at time $t$ in $T D L$.

A conclusion $q$ is strictly provable at time $t$ (figure 4 ) if (1) $q$ is a strict fact valid at time $t$ or (2) there exists a strict rule with conclusion $q[u, v]$ and the instant of time $t$ within $[u, v]$, which rule, (2.1) for all its antecedents $a\left[x_{1}, y_{1}\right]$, there is a time $t^{\prime}$ when they are strictly valid and (2.2) there is no strict negated rule $n s$, attacking rule $r$.

Defeasible derivations have an argumentation like structure [4]: firstly, we choose a supported rule having the conclusions $q$ we want to prove, secondly we consider all the possible counterarguments against $q$, and finally we rebut all 


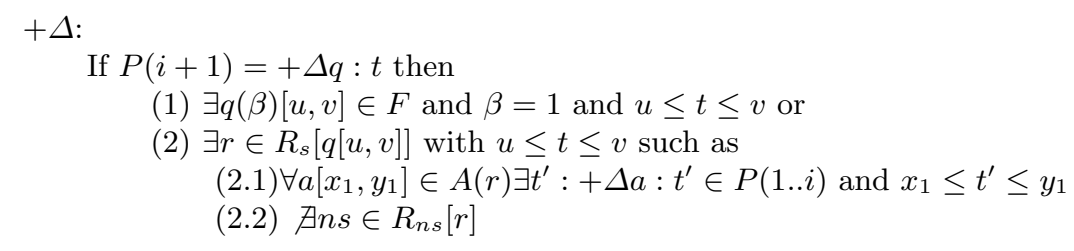

Fig. 4. Definite proof for the consequent $q$ at time $t$ for the rigorous agent.

the above counterarguments showing that, either some of their premises do not hold, or the rule used for its derivation is weaker than the rule supporting the initial conclusion $q$. The sentence $q$ is defeasibly provable at time $t$ (figure 5 ) if

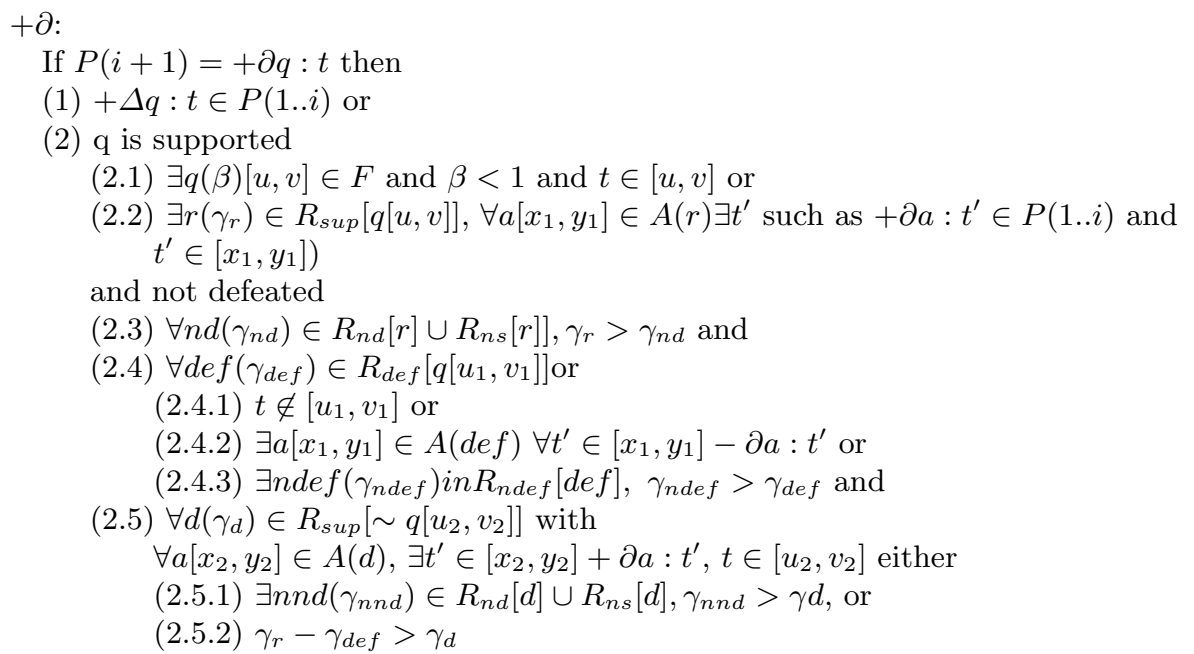

Fig. 5. Defeasible derivation of consequence $q$ at time $t$ for the rigorous agent.

(1) it is strictly provable at $t$, or (2) there is a valid support for $q$ either (2.1) it is a defeasible fact valid at $t$, or (2.2) there exists a rule with all premises valid sustaining that conclusion $q$ and it is not defeated by (2.3) a negated rule with a stronger certainty factor, or (2.4) by an undercutting defeater def where (2.4.1) time $t$ is not within the validity interval of the defeater, or (2.4.2) the defeater has an antecedent $a$ which cannot be derived, or (2.4.3) there exists a negated defeater stronger than def, and (2.5) for all valid rebuttal defeaters $d$ either (2.5.1) there is a negated rule which defeats $d$ or (2.5.2) the support for conclusion $q$ after it is attacked by the undercutter defeaters remains stronger than all the valid rebuttal defeaters. The strict order relation in (2.3), (2.4.3), and (2.5.2) provides a skeptical reasoning mechanism, meaning that none of $q: t$ and $\sim q: t$ 
is derived when they have equal support. Allowing the ambiguity propagation increases the number of inferred conclusions, useful in the argumentation process of ODR systems oriented towards solution rather than finding the degree of guilt.

\subsection{Attitude Component}

The attitude component defines the argumentative attitude of an agent towards other participants, making a distinction between the agent's private collection of arguments and its public uttered sentences. We adapt the claim-attitude and concede-attitude [9], defining the level of proof sufficient to convince the opponent that a given sentence is true, to our defeasible formalism.

The following standards of proofs from current legal practice are modeled: scintilla of evidence, reasonable suspicion ${ }^{4}$, preponderance of evidence ${ }^{5}$, clear and convincing evidence, and beyond reasonable doubt ${ }^{6}$.

Definition. Claim-attitude at time $t$

- A confident agent can claim any sentence $q: t$ for which there is a valid support rule $r \in R_{\text {sup }}$ (scintilla of evidence).

- A careful agent can claim any proposition $q: t$ if there is no valid rebuttal defeater sustaining the opposite sentence $\neg q: t$ (reasonable suspicion).

- A precaution agent can claim any proposition $q: t$ if there is no valid rebuttal or undercutting defeater for the opposite sentence $\neg q: t$ (preponderance of evidence).

- A thoughtful agent can claim any proposition $q: t$ for which it can construct a defeasible proof $+\partial q: t$ (clear and convincing evidence).

- A strict agent can claim any proposition $q: t$ for which it can construct a definite proof $+\Delta q: t$ according to its theory (beyond reasonable doubt).

Definition. Concede-attitude at time $t$

- A credulous agent can concede to any sentence $q: t$ for which it has a valid support rule $r \in R_{\text {sup }}$ (scintilla of evidence).

- $A$ cautious agent can concede to any proposition $q: t$ if it is not able to provide a stronger rebuttal defeater for the opposite sentence $\neg q: t$ (reasonable suspicion).

- A vigilant agent can concede to any proposition $q: t$ if it is not able to provide a stronger rebuttal or undercutting valid defeater (preponderance of evidence).

\footnotetext{
${ }^{4}$ Reasonable suspicion is a low standard of proof used to determine whether a brief investigative stop or a brief search by a police officer is warranted.

5 Also known as the "balance of probabilities", this standard is met if the proposition is more likely to be true than not true

6 This means that the proposition must be proved to the extent that there is no "reasonable doubt" in the mind of a reasonable person, such as $90 \%$ certain in the US.
} 
- A skeptical agent can concede only to those propositions $q: t$ for which it can construct a defeasible proof $+\partial q: t$ (clear and convincing evidence).

- A wary agent can concede to any proposition $q: t$ for which it can construct a definite proof $+\Delta q: t$ according to its theory (beyond reasonable doubt).

During the argumentation process, a confident agent might claim any proposition for which it is able to construct an argument (propositions which are not credible can also be uttered). When, for example, the knowledge base of the agent consists of the rules $r_{1}:(0.5): a[1,1] \Rightarrow q[2,2]$, and $r_{2}: b[1,1] \rightarrow \neg q[2,2]$ where $a$ and $b$ are strict valid facts, then it is still presumable for the agent to claim $q$, even if it is aware of the existence of the stronger counterargument $r_{2}$ sustaining the opposite consequent. A careful agent does not communicate a sentence if it is conscious about the validity of a rebuttal defeater, no matter what certainty factor that argument has. Similarly, a precaution agent additionally considers the validity of an undercutter defeater in order to minimize the risk of a potential counterattack from the other disputants. A more critical attitude is the thoughtful one, where an agent will claim propositions for which it is able to construct an acceptable argument, an argument which is defeasibly provable from its knowledge base. A strict agent does not take any risk to be combated in its claims, therefore it conveys only sentences supported by strict inference according to its defeasible theory. The concede-attitudes are used similarly to the claim-attitudes.

\section{Choosing the Proper Strategy}

Various situations might be encountered. (i) The market may have substantial authority, and the same mediation strategy is imposed to all disputants. (ii) Consistent with party autonomy, the agents may settle on different mediation strategies at contracting time or just prior to the arbitration. This approach increases the flexibility and efficiency, because the agents are the ones who know what type of mediation strategy better protects their interests ${ }^{7}$. (iii) All the above mediator's strategies might be used during the resolution process ${ }^{8}$.

In markets where the consumer protection is the main concern, the mediator may provide different interfaces to the disputants. For instance, the persuasion strategy might guarantee high level of protection to the client being irritated by several issues. The strategies may also be correlated to the current dispute: persuasion strategy is connected to cases involving fairness or good faith. Similarly, the persuasion strategy is adequate in the first stage of the dispute, the so called evidential phase, when the factual information is collected.

\footnotetext{
${ }^{7}$ Mediators and arbitrators are humans who might have biases and prejudices. Frequently, the disputants have the opportunity to select the arbitrator who is likely to be sensitive to their predicament.

${ }^{8}$ Most of the human mediators use a form of the co-mediation model. Having two mediators can be an effective way to deal with many different ODR challenges, fitting well to legal systems based on jury.
} 
On the one hand, a probabilistic approach is a good candidate when the dispute process is in its early stages, when there is little information available, and the mediator tries to figure out if the initial claim is warranted ${ }^{9}$. It also may be considered when the information sources are not trust-able. On the other hand, when the process reaches its maturity stage, the irrelevant facts become clear. Therefore, within a fuzzy inference, the unimportant facts do not influence the decision. Legal rules are often open to several interpretations because some terms within legal texts are vague. It is the mediator who gives the appropriate interpretation to terms such as reasonable or sufficient. The agent strategy depends on the active legal doctrines within the market. If the required standard of proof is preponderance of evidence, the probabilistic approach fits better, but when beyond a reasonable doubt doctrine is active, the fuzzy reasoning is appropriate.

The attitude component is relevant in the context of revealing information. Sometimes, the arguments uttered, either fact or rule, represent private information. The agents must assign a utility cost to revealing information, as well as a utility to winning an argument. The strategy depends on the rules of dialog game where the agent participates. When the dialog protocol stipulates that a claim which has been defeated by a party cannot be uttered again, then a strict or thoughtful attitude must be considered. Opposite, a confident attitude is adequate when a party wants to find information, because his opponent defeats the claim by revealing his private arguments.

The relevant question concerns the validity of the semantic model. This question requires empirical evaluations with realistic test cases ${ }^{10}$ in order to choose the best suited defeasible pattern within a particular market. The common disputes are translated into defeasible theories ${ }^{11}$, and the agent's decision is compared with the one given by the human mediator. The highest scored strategy is provided to the disputant who might better anticipate the verdict and the execution timing. The advantage here consists in the fact that judicial cases that are not conforming to a pattern useful in deriving rules, are not treated as noise and removed. Simply, they are considered exceptions and encapsulated as defeaters or strong defeasible rules.

\section{Dispute Resolution Phases}

The client orders a hardware object through a shop-on-line web site (scenario adapted from [11]). The seller has published general contractual conditions on the web site. One of the clauses stipulates that if the product sent is defective, the client has the right to get it repaired or replaced, depending on the seller's choice. After an order is made at $t_{0}$, the seller sends the item. When the client

\footnotetext{
${ }^{9}$ The probable cause doctrine may be invoked which requires a fair probability that a breach took place. Courts vary when determining what constitutes a "fair probability," some say $30 \%$, others $40 \%$, others $51 \%$.

10 See http://www.as.uky.edu/polisci/ulmerproject/index.html for a collection of such a legal dataset.

${ }^{11}$ ILP techniques are available for deriving defeasible theories from legal datasets [10].
} 
receives it at $t_{7}$, he notices both that it does not work and its design was not quite similar to the picture on the web site. The seller accepts that the hardware might be defective, but invokes the mentioned clause. His default choice is to repair the item, but he also proposes to replace the product if the client accepts to pay the transport fee. The client replies that he will only pay half the fee. The client asks an ODR system for arbitration, submitting his argumentation. The seller asks the product to be replaced. The ODR system accepts to lead the arbitration and notifies the seller. The seller accepts and submits his own argumentation.

\subsection{Commencement of Dispute}

A dispute action is commenced by filling a complaint. If minimum of evidence is provided $^{12}$, the mediator takes into consideration the plaintiff's claim. Consequently, a judicial summon is addressed to the defendant. The probabilistic rigorous mediator with a credulous concede attitude is appropriate for this stage. The plaintiff believes with a certainty factor of 0.9 that the picture illustrating the item was irrelevant ( $f_{2}$ in figure 6 ). Considering rule $r_{5}$ such a mediator will prove the + rreplace $: 7$ conclusion with a certainty factor of $0.9 * 0.95=0.855$. Because this value is greater than the threshold of 0.2 , the complaint is accepted and a dispute starts.

\subsection{Discovery}

The discovery is the pre-trial phase in a lawsuit in which each disputant can request evidence from the other party. Under the duty of disclose doctrine, the disputants have the obligation to share their own supporting evidence without being requested to by the other party. Failure to do so can preclude that evidence from being used in trial ${ }^{13}$. Modern dispute resolution strategies try to set the dispute in its early stages. Thus, the discovery phase is meant to clarify what the lawsuit is about, and perhaps to make a party realize it should settle or drop the claim, all before wasting court resources ${ }^{14}$. Because this early phase is mainly about evidence, a probabilistic epistemic mediator is recommended. Also, confident or careful claim attitudes prevail in obtaining information. During this dialog, the following facts become known: the item might be defective (defeasible fact $f_{1}$ has a certainty factor of 0.9 ), and the seller option is to repair the item $\left(f_{3}\right)$. He advocates this through the contractual clauses $r_{1}, r_{2}, r_{3}$, and $r_{4}$, accepted by the buyer when the contract has been signed. The seller proposes to repair the product if the client accepts to pay the transport fee $\left(r_{6}\right)$. The client might agree to pay half the fee $\left(r_{7}\right)$ in order to derive the seller_choice_replace consequent, which is defeated by the seller response $r_{8}$.

12 The claim is supported with $20 \%$ certainty factor.

13 This applies only to evidence that supports their own case, not anything that could harm their case.

14 A procedural rule stipulates that parties have the right to query 25 questions to each other in order to reveal information. 


\subsection{Pre-trial}

The pre-trial represents the last gate-keeping function before trial, answering the question of whether the claim could even go to the arbitration phase. In this stage, the movant can affirmatively negate the claim, whilst the plaintiff may provide different arguments to support the claim. Therefore, a probabilistic persuasion mediator is appropriate in this stage. Because the negation of claims is modeled by rebuttal defeaters, the vigilant concede attitude functioning under the reasonable suspicion doctrine is recommended. The rebuttal defeater $r_{9}$ is conveyed by the defendant who argues that usually he does not replace items to non-premium customers. The probabilistic persuasion mediator will derive the replace conclusion with a certainty factor of 0.985 .

\subsection{Arbitration}

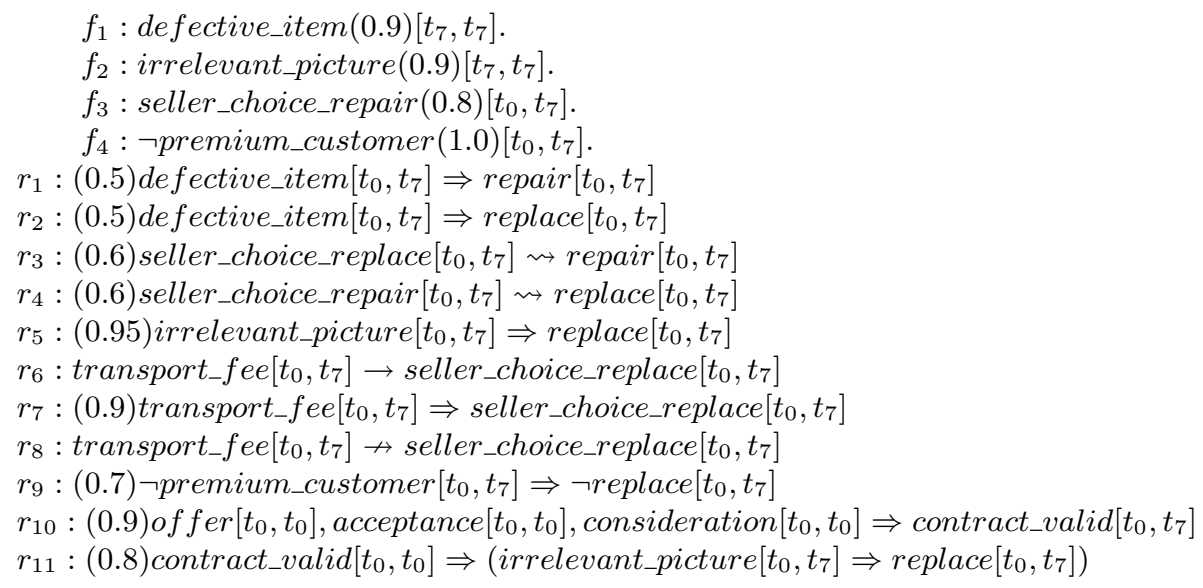

Fig. 6. Sample of arguments collected during the run of a dispute

This phase is the presentation of the evidence gathered during earlier stages (figure 6). In the next step, the mediator decides to which jurisdiction the case belongs and loads the corresponding legal doctrines encapsulated as defeasible theories. He uses both the hard law (enactments, i.e. $r_{10}$ ) and the soft law (usages, customs within the e-market, i.e $r_{11}$ ) to activate the rules or to adjust the certainty factor of the disputants' arguments. As nested rules are allowed in our framework the activation can be done dynamically (rule $r_{11}$ ). Consider that offer, acceptance, and consideration accepted as strict facts, the contract is validated with a certainty factor of $0.9^{15}$. Thus, the dynamic rule

${ }^{15}$ In order to accommodate some exceptions like "the signer is under 18 ". 
is activated with a certainty factor of $\min (0.9,0.8)=0.8$, resulting the rule $r_{5}^{\prime}(0.8)$ : irrelevant_picture $\Rightarrow$ replace, which takes the place of the rule $r_{5}$ in figure 6 . This mechanism provides the mediator the ability to dynamically adjust priorities among rules ${ }^{16}$.

We recommend a fuzzy rigorous mediator with a skeptical concede attitude in order to compute the expected outcome. In the probabilistic approach the claim replace is sustained by the rule $r_{2}$ with 0.5 and by the rule $r_{5}^{\prime}$ with $0.72 * 0.9=$ 0.64. In the fuzzy approach $r_{5}^{\prime}$ supports the consequent with $\min (0.8,0.9)$. According to the fuzzy rigorous mediator $\max (0.5,0.8)-\max (0,6)<\max (0.7)$, therefore the conclusion replace is not derived. Users can also explore hypothetical situations when mediators have different strategies: fuzzy, probabilistic, persuasion or epistemic. How the dispute outcome depends on each defeasible mediator type is shown in figure 7 . Here, in case the ambiguity propagation is enabled, the fuzzy persuasion agent proves the consequent. Users are also able to have dialogs with the system to explore what would happen if some of their claims were rejected or just partially accepted ${ }^{17}$.

\begin{tabular}{|c|c|c|c|}
\hline+ dreplace $: t_{7}$ & Persuasion & Epistemic & Thorough \\
\hline Probabilistic & $N o_{0.5+0.64-0.6<0.7}$ & $N o_{\max (0.5,0.64)<\max (0.6,0.7)}$ & $N o_{\max (0.5,0.64)-0.6<0.7}$ \\
\hline Fuzzy & $N o / Y e s_{0.5+0.8-0.6=0.7}$ & $\operatorname{Nes}_{\max (0,5,0.8)>\max (0.6,0,7)}$ & $N o_{\max (0.5,0.8)-0.6<0.7}$ \\
\hline
\end{tabular}

Fig. 7. Answer for query $+\partial$ replace $: t_{7}$ depends on mediator type.

\subsection{Post-trial}

After the arbitration is done two paths might follow: the enforcement of judgment and appealing the results of the arbitration process. Appealing after the trial may be quite difficult. To facilitate trust in e-commerce, many governments have enacted a norm similar to: "Any item achieved in online transaction can be returned within 15 days, without reason." Under these circumstances, the seller concedes to replace the defective item within 3 days if the client requests this: $r_{20}$ : request $\left[t_{8}, t_{8}\right] \rightarrow$ must_replace_item $\left[t_{8}, t_{10}\right]$. If the client is satisfied this obligation is no longer active: $r_{21}$ : satisfied $\left[t_{8}, t_{1} 0\right] \rightarrow \neg$ must_change_item $[8,10]$. The last role of the system is to monitor contract enactment. This is done simply by trying to prove +2must_change_item : 10 . If the client has asked for replacement and within 3 days he did not get satisfaction, the obligation still stands.

${ }^{16}$ Under most laws, the arbitrator can assign as much probatory force as he believes they deserve, as long as this assessment is not arbitrary [11]. In the long run of ODR it is necessary to create specialized jurisdiction for e-commerce cases, where the certainty factor would be fine tuned according to precedents and mediator experience.

${ }^{17}$ For the scenario in figure 6 if the certainty factor of irrelevant_picture fact is greater than 0.8 a persuasion mediator will infer the replace conclusion. 


\section{Related Work}

The need for computerized mechanisms for decision support comes from well known limits of human knowledge processing. One aim is to provide disputants information about the expected outcome of the resolution process ${ }^{18}$. The other goal is to enrich the mediator's ability to process knowledge and weight arguments. By enhancing the expertise level of the mediator we argue that such decision support system can be looked at as a fourth party as defined in [13].

In the DiaLaw system [14], if the respondent of an argument accepts all the premises of a valid argument, he must also accept the conclusion, in case the respective inductive rule was previously accepted. In our framework, in the light of new information, an undercutting defeater might be used to attack the link between the premises and the consequent. In our view, the existence of a finite set of pre-agreed rules is not feasible for practical applications ${ }^{19}$. Thus, both facts and rules may be dynamically asserted and retracted within the defeasible framework.

In the Carneades argumentation framework [15] three kinds or premises are used: ordinary premises, presumptions, and exceptions, where presumptions are used to model uncertain knowledge. In our approach, the nondeterminacy inherent in the early stages is handled by probabilistic reasoning, whilst defeaters deal with exceptions and information obtained during the resolution process. The framework also deals with information about dialectical status of a sentence: undisputed, at issue, accepted, rejected. We treated this issue elsewhere [16], by defining defeasible commitment machines as a flexible mechanism to adapt the life-cycle of the conveyed facts.

An ODR system was modeled within a multi-agent context by identifying and representing the types of communication between the different actors: parties, mediator, arbitrator, experts, witnesses, ODR-administrator, systemadministrator, visitors [11]. Our approach does not regard the architectural requirements of an ODR system, but rather the reasoning capabilities of the arbitrator.

Rule-based systems are suitable for modeling the logical structure of legislation and are practically successful when the gap between factual and legal language is small and the regulations are uncontroversial, but they fail to model legal argument. Defeasible logic, through its argumentative semantics, overcomes this drawback. It is also adequate in practical applications due to its low complexity [17]. As a simulation tool, the ODR system designer may obtain results

${ }_{18}$ In the negotiation literature this is called BATNA: Know your best alternative to a negotiated agreement [12]

19 By accepting a jurisdiction parties practically agree on a set of legal rules. Through a signed contract, parties agree not only on some contractual clauses, but also regarding several specific doctrines under which that contract is enacted (such as expectation damages, opportunity costs, reliance damages). Due to the open character of both legal rules and contractual clauses, there are situations when supplementary rules have to be considered. 
regarding what types of strategies better suit the e-market or how information sharing can be used to settle the dispute.

The formalization of virtual organizations and contracts based on commitments [18] opens another path for ODR by enabling to capture the social structure. Changes of organizations impose some treatment for the dynamics of enacted contracts.

\section{Conclusions}

There is a strong motivation for the need of ODR systems to reflect different types of argumentation patterns, mainly those models where persuasion can be functionally embedded into negotiation protocols [2]. From the knowledge representation viewpoint the implemented system accommodates temporal defeasible reasoning, nested rules, and a dynamic notion of priority over the rules ${ }^{20}$. From the argumentative semantics viewpoint the system introduces negated rules to model counter-exampls, whilst Pollock's style undercutting defeaters are used to represents exceptions.

We advocate two strong points of this approach: (i) the flexibility of the framework due to the different patterns of weighting arguments and to the property of defeasible logic to model exceptions; (ii) the accordance to legal practice, by establishing a connection between these patterns and disputes phases as they appear in current practice in law. This view on the ODR issue does not insist on the temporal aspects included in the logic. They can be subject to further investigation regarding the application of the framework to contract enactment [18]. Our future work regards also the enrichment of the logical framework with explanation capabilities of the outcome, as a need for the trustworthiness and practical usability in a dispute resolution system.

\section{Acknowledgments}

We are grateful to the anonymous reviewers for useful comments. Part of this work was supported by the grant 27702-990 from the National Research Council of the Romanian Ministry for Education and Research.

\section{References}

1. Tyler, M.C., Bretherton, D.: Seventy-six and counting: An analysis of ODR sites. In: Workshop on Online Dispute Resolution at the International Conference on Artificial Intelligence and Law, Edinburgh, UK (2003) 13-28

2. Walton, D., Godden, D.: Persuasion dialogues in online dispute resolution. Artificial Intelligence and Law 13 (2006) 273-295

3. Rule, C., Friedberg, L.: The appropriate role of dispute resolution in building trust online. Artificial Intelligence and Law 13 (2006) 193-205

${ }^{20}$ A prototype based on LISA (Lisp-based Intelligent Software Agents) is available at http://cs-gw.utcluj.ro/ adrian/tdl.html. 
4. Governatori, G.: Representing business contracts in RuleML. Journal of Cooperative Information Systems 14 (2005)

5. Hage, J.: Law and defeasibility. Artificial Intelligence and Law 11 (2003) 221-242

6. Pollock, J.L.: Defeasible reasoning with variable degrees of justification. Artificial Intelligence 133 (2001) 233-282

7. Pollock, J.L.: How to reason defeasibly. Artificial Intelligence 57 (1992) 1-42

8. Prakken, H.: A study of accrual of arguments, with applications to evidential reasoning. In: 10th International Conference on Artificial Intelligence and Law, New York, NY, USA (2005) 85-94

9. Parsons, S., Wooldridge, M., Amgoud, L.: Properties and complexity of some formal inter-agent dialogues. Journal of Logic and Computation 13 (2003) 347376

10. Johnston, B., Governatori, G.: An algorithm for the induction of defeasible logic theories from databases. In: Australasian Database Conference. (2003) 75-83

11. Bonnet, V., Boudaoud, K., Gagnebin, M., Harms, J., Schultz, T.: Online dispute resolution systems as web services. ICFAI Journal of Alternative Dispute 3 (2004) $57-74$

12. Bellucci, E., Lodder, A.R., Zeleznikow, J.: Integrating artificial intelligence, argumentation and game theory to develop an online dispute resolution environment. In: 16th International Conference on Tools with Artificial Intelligence, IEEE Computer Society (2004) 749-754

13. Katsh, E., Rifkin, J.: Online Dispute Resolution: Resolving Conflicts in Cyberspace. John Wiley (2001)

14. Lodder, A.: DiaLaw: On Legal Justification and Dialogical Models of Argumentation. Kluwer, Dordrecht (1999)

15. Gordon, T., Walton, D.: The Carneades argumentation framework: Using presumptions and exceptions to model critical questions. In: 1st International Conference on Computational Models of Argument, Amsterdam, IOS Press (2006) 208-219

16. Letia, I.A., Groza, A.: Running contracts with defeasible commitment. In Moonis, A., Dapoigny, R., eds.: Advances in Applied Artificial Intelligence. LNCS 4031. Springer (2006) 91-100

17. Maher, M.J.: Propositional defeasible logic has linear complexity. Theory and Practice of Logic Programming 1 (2001) 691-711

18. Udupi, Y.B., Singh, M.P.: Contract enactment in virtual organizations: A commitment-based approach. In: 21st National Conference on Artificial Intelligence, AAAI (2006) 722-727 